\title{
Investigating Different DNA Methylation Patterns at the Resolution of Methylation Haplotypes
}

\author{
Xiaoqing Peng ${ }^{1 *}$, Yiming $\mathrm{Li}^{1}$, Xiangyan Kong ${ }^{2}$, Xiaoshu Zhu ${ }^{3}$ and Xiaojun Ding ${ }^{3 *}$ \\ ${ }^{1}$ Center for Medical Genetics \& Hunan Key Laboratory of Medical Genetics, School of Life Sciences, Central South \\ University, Changsha, China, ${ }^{2}$ School of Computer Science and Engineering, Central South University, Changsha, China, \\ ${ }^{3}$ School of Computer Science and Engineering, Yulin Normal University, Yulin, China
}

OPEN ACCESS

Edited by:

Wei Lan,

Guangxi University, China

Reviewed by:

Hao Lin,

University of Electronic Science and

Technology of China, China

Leyi Wei,

Shandong University, China

*Correspondence:

Xiaoqing Peng

xqpeng@csu.edu.cn

Xiaojun Ding

ding.xiaojun@foxmail.com

Specialty section:

This article was submitted to

Computational Genomics,

a section of the journal

Frontiers in Genetics

Received: 19 April 2021

Accepted: 01 June 2021

Published: 28 June 2021

Citation:

Peng $X, L i Y$, Kong $X$, Zhu $X$ and Ding $X$ (2021) Investigating Different

DNA Methylation Patterns at the Resolution of Methylation Haplotypes.

Front. Genet. 12:697279.

doi: $10.3389 /$ fgene.2021.697279
Different DNA methylation patterns presented on different tissues or cell types are considered as one of the main reasons accounting for the tissue-specific gene expressions. In recent years, many methods have been proposed to identify differentially methylated regions (DMRs) based on the mixture of methylation signals from homologous chromosomes. To investigate the possible influence of homologous chromosomes on methylation analysis, this paper proposed a method (MHap) to construct methylation haplotypes for homologous chromosomes in $\mathrm{CpG}$ dense regions. Through comparing the methylation consistency between homologous chromosomes in different cell types, it can be found that majority of paired methylation haplotypes derived from homologous chromosomes are consistent, while a lower methylation consistency was observed in the breast cancer sample. It also can be observed that the hypomethylation consistency of differentiated cells is higher than that of the corresponding undifferentiated stem cells. Furthermore, based on the methylation haplotypes constructed on homologous chromosomes, a method (MHap_DMR) is developed to identify DMRs between differentiated cells and the corresponding undifferentiated stem cells, or between the breast cancer sample and the normal breast sample. Through comparing the methylation haplotype modes of DMRs in two cell types, the DNA methylation changing directions of homologous chromosomes in cell differentiation and cancerization can be revealed. The code is available at: https://github.com/xqpeng/MHap_DMR.

Keywords: methylation haplotype, differentially methylated region, cell differentiation, homologous chromosomes, methylation consistency, hypomethylation consistency

\section{INTRODUCTION}

In recent years, the revealing of the mechanisms behind the diseases has been performed from different angles, such as mutated genes, altered DNA methylation (Eden et al., 2003; Baylin, 2005), non-coding RNAs (Yan et al., 2017, 2018; Chen et al., 2019; Lan et al., 2020), microbes (Yan et al., 2019, 2021), etc. Differentially methylated regions (DMRs) are the main explanation accounting for the diversity of gene expression in different cell types in a body. Differentiation-associated differential methylation profiles were observed on cell types under different stages of development and differentiation (Laurent et al., 2010). Recent studies show that altered DNA methylation has a very close relationship with diseases. In cancer genomes, the promoter regions of tumor suppressor genes are altered to be hypermethylated (Baylin, 2005), while the promoter regions of tumor genes 
are altered to be hypomethylated (Eden et al., 2003). Identifying DMRs can promote revealing the mechanisms in tissuespecific/diseases-specific gene expression (Scott et al., 2020) and tissue-specific DMRs can be used as feature markers in identifying the tissues-of-origin of cfDNAs in noninvasive diagnosis (Hu et al., 2019; Xiaoqing et al., 2020).

Infinium HumanMethylation450 BeadChip and Infinium MethylationEPIC BeadChip provide a convenient way to measure the methylation levels of $\mathrm{CpG}$ sites in $\mathrm{CpG}$ islands and gene regions. In BreadChips, the methylation level of a certain CpG site is estimated by using the ratio of intensities between methylated and unmethylated alleles. In recent years, due to the development of sequencing technology, bisulfite sequencing (BSSeq) makes to reveal the methylation status of each cytosine site on a read become possible. The numbers of methylated cytosines and unmethylated ones of each cytosine site can be measured, respectively. Recently, by using deep-learning, DNA methylation status of each cytosine site can be deduced from Nanopore sequencing reads (Ni et al., 2019). In both BeadChip and BS-Seq, molecules derived from two homologous chromosomes are not discriminated and are always processed together.

Based on the methylation profiles extracted from BeadChips or BS-Seq data, many methods have been proposed to identify DMRs in different tissues or cell types. These methods can be roughly divided into two categories: differentially methylated cytosine site (DMC)-based methods and candidate region-based methods. In DMC based methods, methylation levels of $\mathrm{CpG}$ sites can be calculated based the raw methylation information of CpG sites (Catoni et al., 2018; Condon et al., 2018; Xu et al., 2020), estimated by beta-binomial distribution considering the biological variances and sample variances (Feng et al., 2014; Park et al., 2014; Lea et al., 2015; Wu et al., 2015; Park and Wu, 2016; Wen et al., 2016) or estimated by considering the spatial correlation (Hansen et al., 2012; Hebestreit et al., 2013; Wu et al., 2015; Sun and Yu, 2016). Then, DMCs are identified and DMRs are formed by merging the neighboring DMCs satisfying some defined criteria, such as DMCs with $p$-values less than a certain threshold, the distance between the DMCs less than a cutoff value, and the minimum number of DMCs required in a region.

In candidate region-based methods, there are two types of candidate regions, including sample-dependent candidate regions and sample-independent ones. The sample-independent candidate regions are predefined on the genome with a fixedsize or sliding window (Stockwell et al., 2014; Wang et al., 2015; Catoni et al., 2018). The sample-dependent candidate regions are generated according to the coverage, the depth of $\mathrm{CpG}$ sites, the methylation levels of CpG sites in samples, and the methylation changes of $\mathrm{CpG}$ sites among multi-samples. Then DMRs are identified by comparing the methylation of regions among different samples (Su et al., 2012; Lokk et al., 2014; Liu et al., 2015; Jühling et al., 2016; Gomez et al., 2019).

As we known, the allele-specific methylation is a special phenomenon of DNA methylation, which is that the methylation of an allele on two homologous chromosomes is not consistent. With the development of high-throughput sequencing technology, the region capture based sequencing and the genome-wide sequencing have been widely used for detecting allele-specific methylation sites. Some strategies and algorithms also contribute to improve the identification of allele-specific methylation (Cheung et al., 2017; Abante et al., 2020). However, the research on identifying allele-specific methylation is limited to the alleles, and the influence of homologous chromosomes on methylation analysis should be investigated genome wide.

In the methods of identifying DMRs, the reads from homologous chromosomes are processed together, and the methylation levels of CpG sites are calculated based on the mixture of reads from homologous chromosomes. The influence of homologous chromosomes on methylation analysis was not considered and investigated. To investigate the possible influence of homologous chromosomes on methylation analysis, we construct methylation haplotypes for homologous chromosomes on the sample-independent candidate regions. Then the methylation consistency of paired methylation haplotypes from homologous chromosomes in different cell types is compared. Further, DMRs are identified at the resolution of methylation haplotypes. The proposed method in this paper not only can be applied to methylation analysis, but also can provide a clear explanation for the methylation difference at the resolution of methylation haplotypes.

\section{MATERIALS AND METHODS}

In this paper, two methods, MHap and MHap_DMR, are proposed to construct methylation haplotypes and identify DMRs based on methylation haplotypes, respectively. MHap is a method for constructing methylation haplotypes, which consists of four steps. Firstly, it generates sample-independent candidate regions based on genomic information, such as $\mathrm{CpG}$ islands and CpG dense regions. Then, for the BS-seq data of each sample, it classifies CpG sites into homozygous and heterozygous ones, and then constructs methylation haplotypes for each candidate region. Finally, the paired methylation haplotypes of homologous chromosomes are represented by methylation haplotype modes (MHMs). MHap_DMR is the method designed to identify DMRs based on methylation haplotypes. The framework of MHap and MHap_DMR is shown in Figure 1 and the detail of each step in the proposed methods will be described in the following subsections.

\subsection{Materials}

To investigate the influence of homologous chromosomes on methylation analysis, 12 WGBS datasets of 10 different tissues/cell types are involved in this study, including two lower leg skin samples and two tibial nerver samples downloaded from the ENCODE project (The ENCODE Project Consortium, 2012) (access sample id: ENCSR930WUY, ENCSR128RMY, ENCSR752OCM, and ENCSR658MZU), breast cancer sample and normal breast sample in the GEO database under the accession number GSE29069 (Hon et al., 2012), adipose-derived stem (ADS) cells and mature fat cells (adipocytes derived from the ADS cells) in the NCBI SRA database under the accession number SRA023829.2 (Lister et al., 2011), embryonic stem cells (hESCs) and foreskin fibroblasts (hESC-Fibro cells) in the GEO database under the accession number GSE19418 (Laurent et al., 


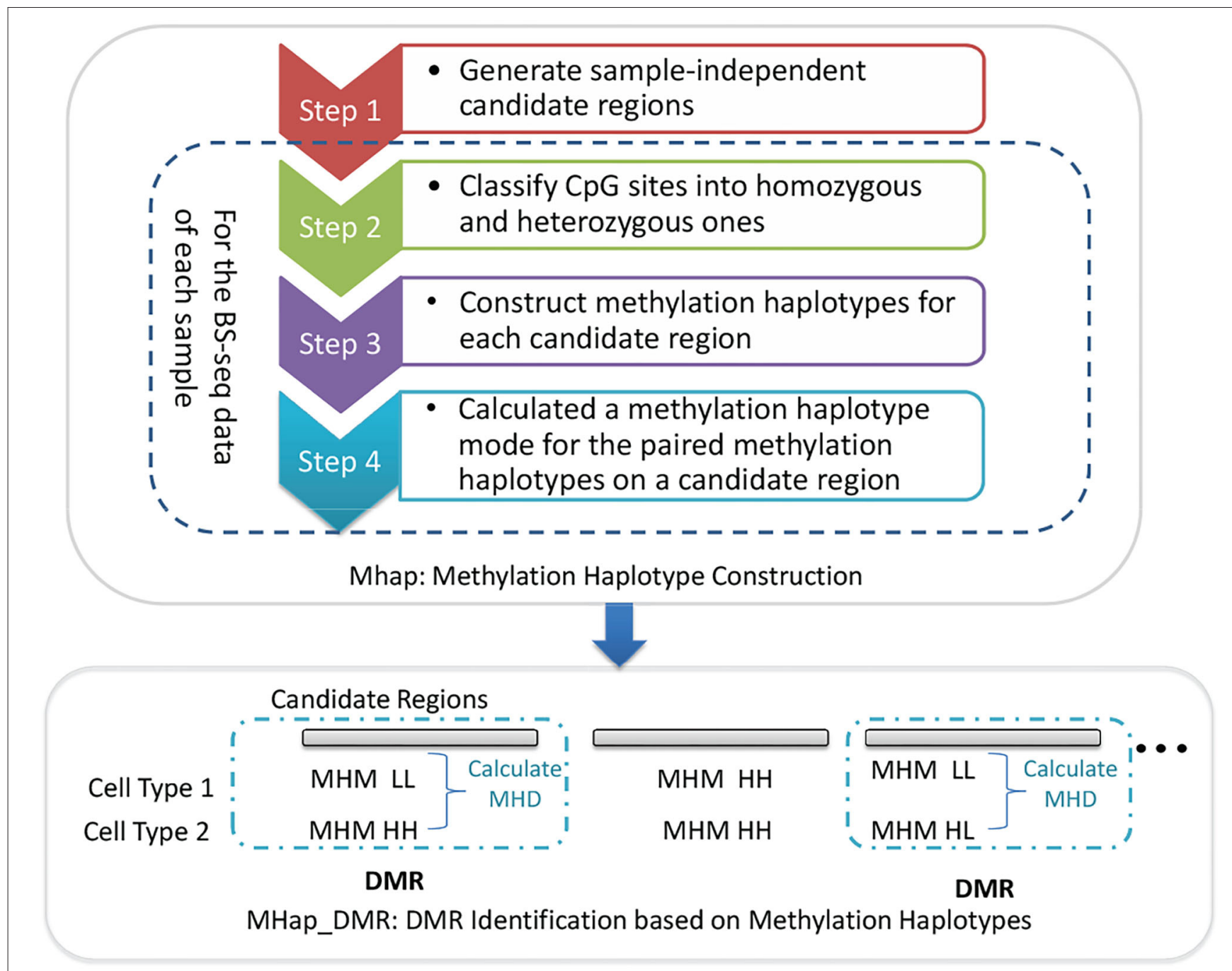

FIGURE 1 | The framework of MHap and MHap_DMR.

2010), mature B cells and hematopoietic stem cells in the GEO database under the accession number GSE31971 (Hodges et al., 2011). The WGBS datasets were aligned to the human reference genome (hg38) and the methylation statuses of cytosines on reads were called by using Bismark (Krueger and Andrews, 2011).

\subsection{MHap: Methylation Haplotype Construction}

Due to the limited read lengths and the uneven distribution of CpG sites, it is challenging to construct two complete methylation haplotypes for two homologous chromosomes. Thus, sample-independent candidate regions are predefined on CpG dense regions, and methylation haplotypes are constructed for homologous chromosomes in these regions. MHap is proposed to construct methylation haplotypes for homologous chromosomes based on the overlapping methylation statuses of heterozygous methylated $\mathrm{CpG}$ sites on reads. The details of MHap is described as following.

\subsubsection{Generate Sample-Independent Candidate Regions}

MHap generates sample-independent candidate regions based on the $\mathrm{CpG}$ island information and the distance between neighboring $\mathrm{CpG}$ sites. In order not to hide local methylation signals, $\mathrm{CpG}$ islands are usually divided into a number of candidate regions, each of which contains at least $7 \mathrm{CpG}$ sites. For other regions with densely located CpG sites, a distance-based clustering algorithm is applied to generating candidate regions, which contains at least $7 \mathrm{CpG}$ sites also and the distances between neighboring $\mathrm{CpG}$ sites are not $>20$ bp. As shown in Table 1, for each chromosome, the number of candidate regions and the corresponding averages of $\mathrm{CpG}$ numbers and region lengths are listed. Then, MHap will construct methylation haplotypes for 
TABLE 1 | The number, the average number of CpGs, and the length of candidate regions in each chromosome.

\begin{tabular}{|c|c|c|c|}
\hline Chromosome & $\begin{array}{c}\text { Num. of } \\
\text { candidate regions }\end{array}$ & $\begin{array}{l}\text { Ave. Num. of } \\
\text { CpGs }\end{array}$ & $\begin{array}{c}\text { Ave. length of } \\
\text { candidate regions }\end{array}$ \\
\hline chr1 & 26,643 & 10.48 & 92.11 \\
\hline chr2 & 20,446 & 10.46 & 91.71 \\
\hline chr3 & 14,013 & 10.46 & 93.08 \\
\hline chr4 & 13,316 & 10.49 & 95.06 \\
\hline chr5 & 14,411 & 10.46 & 93.70 \\
\hline chr6 & 14,378 & 10.49 & 93.86 \\
\hline chr7 & 16,809 & 10.42 & 92.33 \\
\hline chr8 & 12,429 & 10.43 & 92.64 \\
\hline chr9 & 13,798 & 10.44 & 91.80 \\
\hline chr10 & 13,482 & 10.46 & 91.90 \\
\hline chr11 & 14,194 & 10.43 & 91.38 \\
\hline chr12 & 13,107 & 10.42 & 93.56 \\
\hline chr13 & 7,503 & 10.41 & 93.78 \\
\hline chr14 & 9,217 & 10.44 & 91.07 \\
\hline chr15 & 9,173 & 10.49 & 89.84 \\
\hline chr16 & 14,837 & 10.36 & 91.21 \\
\hline chr17 & 17,285 & 10.45 & 92.24 \\
\hline chr18 & 6,419 & 10.55 & 92.72 \\
\hline chr19 & 20,663 & 10.51 & 95.05 \\
\hline chr20 & 8,844 & 10.40 & 90.39 \\
\hline chr21 & 5,597 & 10.70 & 92.66 \\
\hline chr22 & 8,153 & 10.38 & 87.39 \\
\hline $\operatorname{chrX}$ & 10,687 & 10.42 & 97.36 \\
\hline chrY & 1,982 & 10.31 & 103.24 \\
\hline
\end{tabular}

homologous chromosomes on these candidate regions. of the candidate regions.

\subsubsection{Classify CpG Sites Into Homozygous and Heterozygous Ones}

The flow char of classifying CpG sites into homozygous and heterozygous ones is illustrated as in Figure 2. For each sample, the reads falling in candidate regions are collected. In these candidate regions, firstly, CpG sites with depth less than a threshold $T h_{d p}$ are filtered out. Then the remaining CpG sites are classified into homozygous sites and candidate heterozygous sites(CHSs) based on the types of methylation statuses and the corresponding depths. If a CpG site has only one methylation status with depth not less than $T h_{d p}$, it is considered as a homozygous site. If it has two methylation statuses and the depth of each status is not less than half of $T h_{d p}$, it is considered as a CHS.

Due to the sequencing errors and the bisulfite conversion rates, the identified CHSs inevitably contain false-positives. The joint methylation statuses of neighboring CHSs on the same reads can help to distinguish true-positives from false-positives. Thus, the joint methylation statuses of two neighboring CHSs on the covering reads are extracted and can be represented as 00/11/01/10 patterns. In MHap, the frequency of each pattern on two neighboring CHSs is calculated, and patterns with frequency $<2$ are filtered. Then, one or two true-positive patterns are identified according to the ratios of the corresponding frequencies to the total frequency of all patterns or to the maximum frequency. If there is a pattern with the maximum frequency among other patterns and the ratio of its frequency to the total frequency of all patterns is above a threshold (recommended as 0.6), it is considered as the only one truepositive pattern on the two neighboring CHSs. Otherwise, if there are two patterns with higher frequencies than other patterns and the ratio of the second maximum frequency to the first maximum frequency is not less than a threshold (recommended as 0.4 ), it is considered that there are two true-positive patterns on the two neighboring CHSs. Then two neighboring CHSs are reclassified into homozygous or heterozygous ones based on the true-positive patterns.

Pairs of neighboring CHSs are processed sequentially. Assume there are three successive CHSs $(u, v, w)$. During the processing of two successive pairs $(u, v)$ and $(v, w)$, the unbalance join depths may result in a conflict on the classification of the overlapped CHS v. To handle with this conflict, a confidence score is calculated for each pair of neighboring CHSs, computed as the ratio of the total frequency of true-positive patterns on two sites to the maximum depth among three $\mathrm{CpG}$ sites, as defined in Equation (1). If $\operatorname{conf}(u, v)>=\operatorname{conf}(v, w)$, the class of $v$ will be not changed, and the class of $w$ will be determined based on the joint methylation statuses of $(v, w)$ with the given class of $v$. If $\operatorname{conf}(u, v)<\operatorname{conf}(v, w)$, the class of $v$ will be revised based on the true-positive patterns of $(v, w)$.

$$
\operatorname{conf}(u, v)=\frac{\sum_{p}^{p \in T P} f(p)}{\max (d(u), d(v), d(w))}
$$

where TP denotes the set of true-positive patterns of $(u, v), f(p)$ denotes the frequency of pattern $p$, and $d(u), d(v)$, and $d(w)$ denote the depths of $u, v$, and $w$, respectively.

\subsubsection{Construct Methylation Haplotypes for Each Candidate Region}

After classifying CpG sites into homozygous and heterozygous ones, the skeletons of two methylation haplotypes are constructed by linking the patterns of neighboring heterozygous sites sequentially. Then, a pair of methylation haplotypes are constructed by padding the homozygous $\mathrm{CpG}$ sites into the skeletons.

\subsubsection{Definition of Methylation Haplotype Mode}

Each methylation haplotype can be represented by a $0-1$ string. To simplify the comparison between methylation haplotypes, each methylation haplotype is converted into a label based on its $0-1$ string, defined in Equation (2). Then, two labels of the paired methylation haplotypes on a candidate region, denoted as $L L, H L, L N, L M, N N, M M, M N, H N, H M$ or $H H$, are termed as a methylation haplotype mode (MHM). 


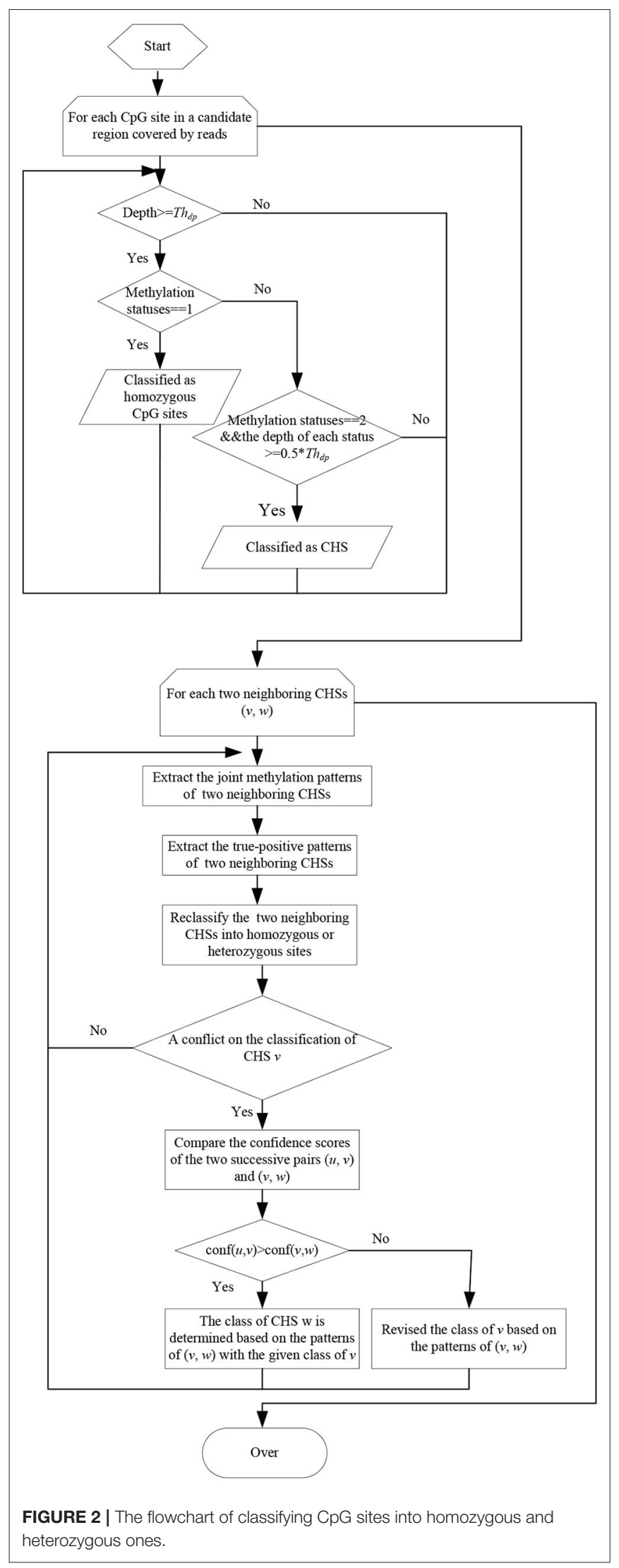

TABLE 2 | Statistics of candidate regions with methylation haplotypes in different samples.

\begin{tabular}{lccc}
\hline Sample & $\begin{array}{c}\text { Num. of } \\
\text { candidate regions } \\
\text { with VMHs }\end{array}$ & $\begin{array}{c}\text { Ave. Num. of } \\
\text { CpG sites }\end{array}$ & $\begin{array}{c}\text { Ave. Num. of } \\
\text { covered CpG }\end{array}$ \\
\hline $\begin{array}{l}\text { Mature fat cells } \\
\text { Adipose-derived }\end{array}$ & 249,253 & 10.51 & 5.91 \\
stem cells & 256,671 & 10.51 & 6.08 \\
Breast cancer & 233,973 & & 6.69 \\
sample & & 10.49 & 6.22 \\
Normal breast & 223,692 & 10.49 & 6.38 \\
sample & & & \\
Hematopoietic stem & 172,536 & 10.44 & 5.20 \\
cells & & 10.44 & 6.05 \\
Mature B cells & 138,053 & 10.63 & \\
Embryonic stem & 220,970 & & 6.10 \\
cells & & 10.66 & 8.67 \\
Foreskin fibroblasts & 213,317 & 10.30 & 8.96 \\
Lower_leg_skin_1 & 228,263 & 10.36 & 8.61 \\
Lower_leg_skin_2 & 244,369 & 10.36 & \\
Tibial_nerve_1 & 239,034 & 10.31 & \\
Tibial_nerve_2 & 225,728 & & \\
\hline
\end{tabular}

$$
\operatorname{Label}(s)=\left\{\begin{array}{l}
L, \text { ifMH}(s) \leq 0.25 \\
N, \text { elseifMH(s) } \leq 0.5 \\
M, \text { elseifMH(s) } \leq 0.75 \\
H, \text { else }
\end{array}\right.
$$

where $M H(s)=\frac{\sum_{i=1}^{\operatorname{len}(s)}\left(s_{i}-0\right)}{\operatorname{len}(s)}, s$ represents the $0-1$ string of a methylation haplotype, len $(s)$ represents the length of $s$, and $s_{i}$ is the $i$-th character in $s$.

\subsection{Map_DMR: DMR Identification Based on Methylation Haplotypes}

Based on the MHMs of each candidate region among different samples, MHap_DMR identifies DMRs by comparing the MHMs directly. If the MHMs are identical, the candidate region is considered as a non-DMR. Otherwise, a methylation haplotype difference (MHD) between a pair of samples or groups is calculated, defined as in Equation (3). Then, the methylation difference among multi groups on the region can be defined as the maximum MHD among pairs of groups.

$$
\begin{aligned}
M H D\left(g_{i}, g_{j}\right)= & \max \left(a b s\left(M H\left(g_{i 1}\right)-M H\left(g_{j 1}\right)\right), a b s\left(M H\left(g_{i 2}\right)\right.\right. \\
& \left.\left.-M H\left(g_{j 2}\right)\right)\right)
\end{aligned}
$$

where $g_{i}$ and $g_{j}$ denote group $i$ and $j, g_{i 1}$ and $g_{j 1}$ denote the $0-1$ strings of methylation haplotypes with higher $M H$ values in $g_{i}$ and $g_{j}$, respectively, and $g_{i 2}$ and $g_{j 2}$ denote the $0-1$ 


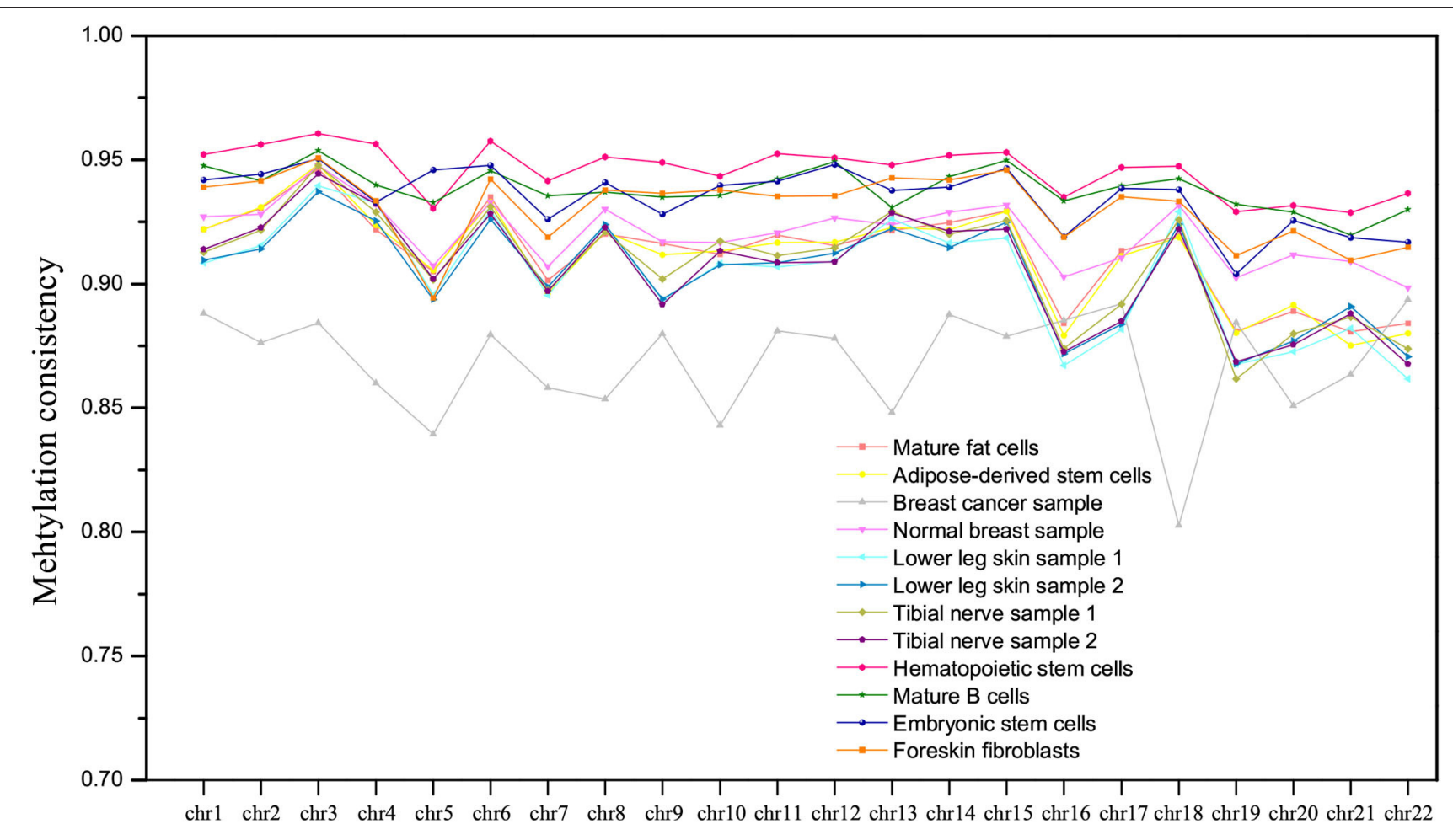

Chromosome

FIGURE 3 | The comparison of methylation consistency of homologous chromosomes in different tissues/cell types.

strings of methylation haplotypes with lower $M H$ values in $g_{i}$ and $g_{j}$, respectively.

To investigate the influence of homologous chromosomes on methylation analysis, we applied MHap to construct methylation haplotypes for 12 WGBS datasets of 10 different tissues/cell types. MHap constructs methylation haplotypes for each sample based on the alignment file and candidate regions. Methylation haplotypes covering more than $3 \mathrm{CpG}$ sites are defined as valid methylation haplotypes (VMHs). Table 2 lists the number of candidate regions with VMHs contained by each sample. It can be observed that the average number of $\mathrm{CpG}$ sites in these candidate regions is $>10$, and the average number of covered CpG sites in VMHs is ranging from 5.9 to 8.9.

\section{RESULT}

\subsection{Majority of Methylation Haplotypes Are Consistent Between Homologous Chromosomes}

MHMs $H H$ and $L L$ denote that the paired methylation haplotypes of two homologous chromosomes are simultaneously hypermethylated $(H H)$ or hypomethylated $(L L)$. Both the $H H$ and $L L$ are considered as consistent MHMs. Then, the methylation consistency between two homologous chromosomes in a sample can be defined as the ratio of the number of CpGs in VMHs with consistent MHMs to that in all VMHs.

The methylation consistency of homologous autosomes in different tissues/cell types is compared, as shown in Figure 3. For normal tissues or cell types, the methylation consistency is above $90 \%$ on average, especially in hematopoietic stem cells. A lower methylation consistency can be observed in the breast cancer sample, which is about $86 \%$ on all the homologous chromosomes.

The methylation consistency of chromosome $\mathrm{X}$ indicates the gender of a sample. In Figure 4, it can be observed that three samples with methylation consistency above $94 \%$ are derived from male, while samples with methylation consistency ranging from 54 to $72 \%$ are derived from female which is much lower than that of other homologous autosomes. It coincides with the previous studies that the methylation between two homologous chromosome $\mathrm{X}$ in female are different, one of which is inactive and highly methylated (Mohandas et al., 1981; Goto and Monk, 1998).

Further, we compared the hypomethylation consistency in different samples. The hypomethylation consistency between two homologous chromosomes in a sample can be defined as the ratio of the number of CpGs in VMHs with consistent MHM $L L$ to that in all VMHs. From Figure 5, we can observe that the hypomethylation consistency of derived cells is higher than that of the corresponding undifferentiated stem cells, which is consistent with the former studies that methylation decrease with 


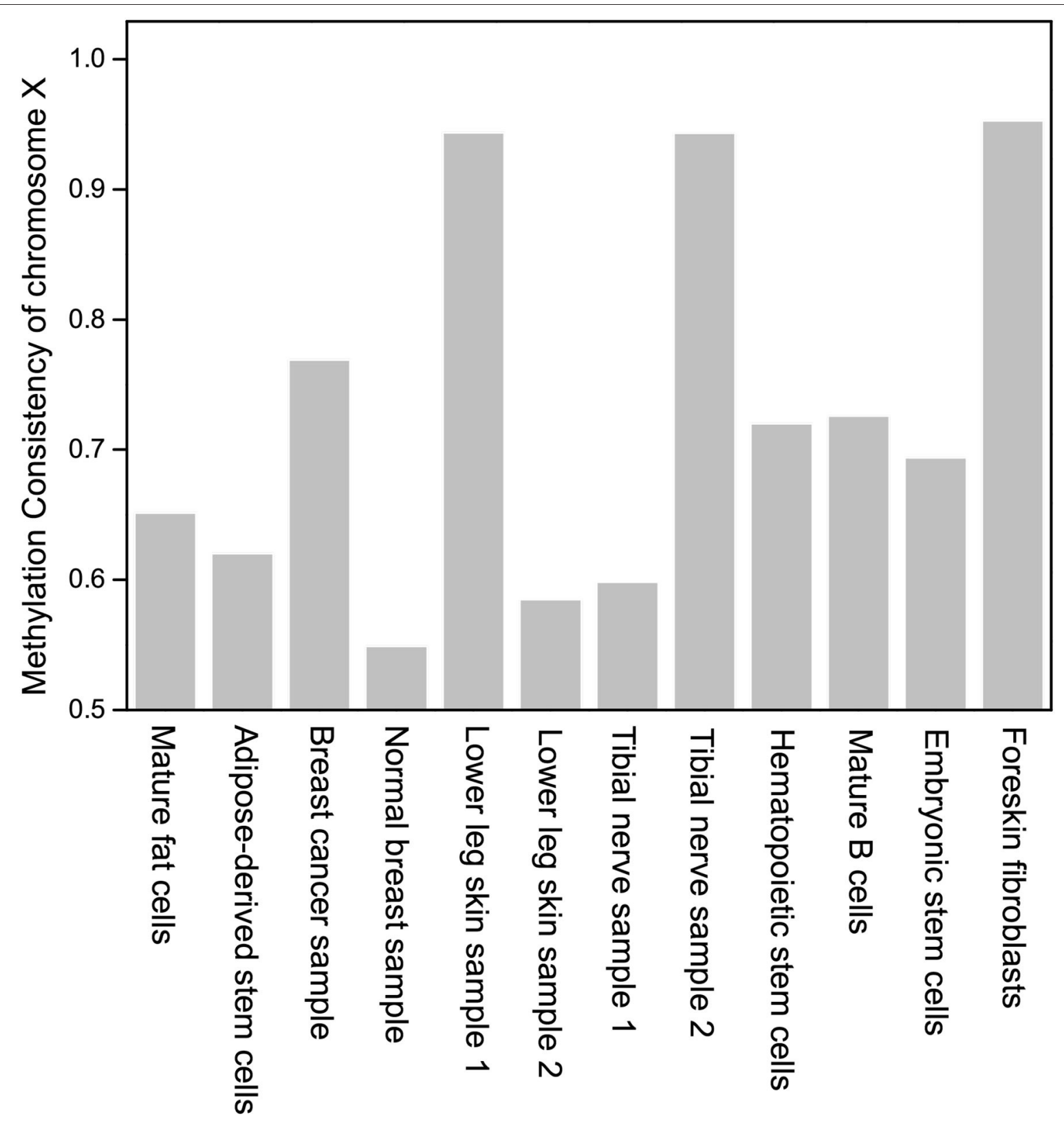

FIGURE 4 | The comparison of methylation consistency of chromosome $\mathrm{X}$ in different samples.

the degree of differentiation increased (Laurent et al., 2010). In Figure 5, we can find that the mature fat cells are more hypomethylated than adipose-derived stem cells, mature B cells are more hypomethylated than hematopoietic stem cells, and foreskin fibroblasts are more hypomethylated than embryonic stem cells. It is also noted that the hypomethylation consistency of breast cancer sample is much lower than that of normal breast sample on homologous chromosome.

In addition, it is interesting to observe that the tissues/cell types can be roughly clustered into three groups according to the hypomethylation consistency, as shown in Figure 5. Lower leg skin and tibial nerve have similar hypomethylation consistency and they belong to the ectoderm. The hypomethylation consistency of mature fat cells, adipose-derived stem cells, mature $\mathrm{B}$ cells, hematopoietic stem cells and the normal breast sample are similar, and these tissues/cell types belong to the mesoderm. The hESCs and hESC-Fibro cell types have high hypomethylation consistency in chromosomes, which are higher than that of other tissues/cell types.

\subsection{Identifying DMRs Between Two Samples}

MHap_DMR was applied to identify DMRs in four pairs of samples, including breast cancer vs. normal breast, mature fat 


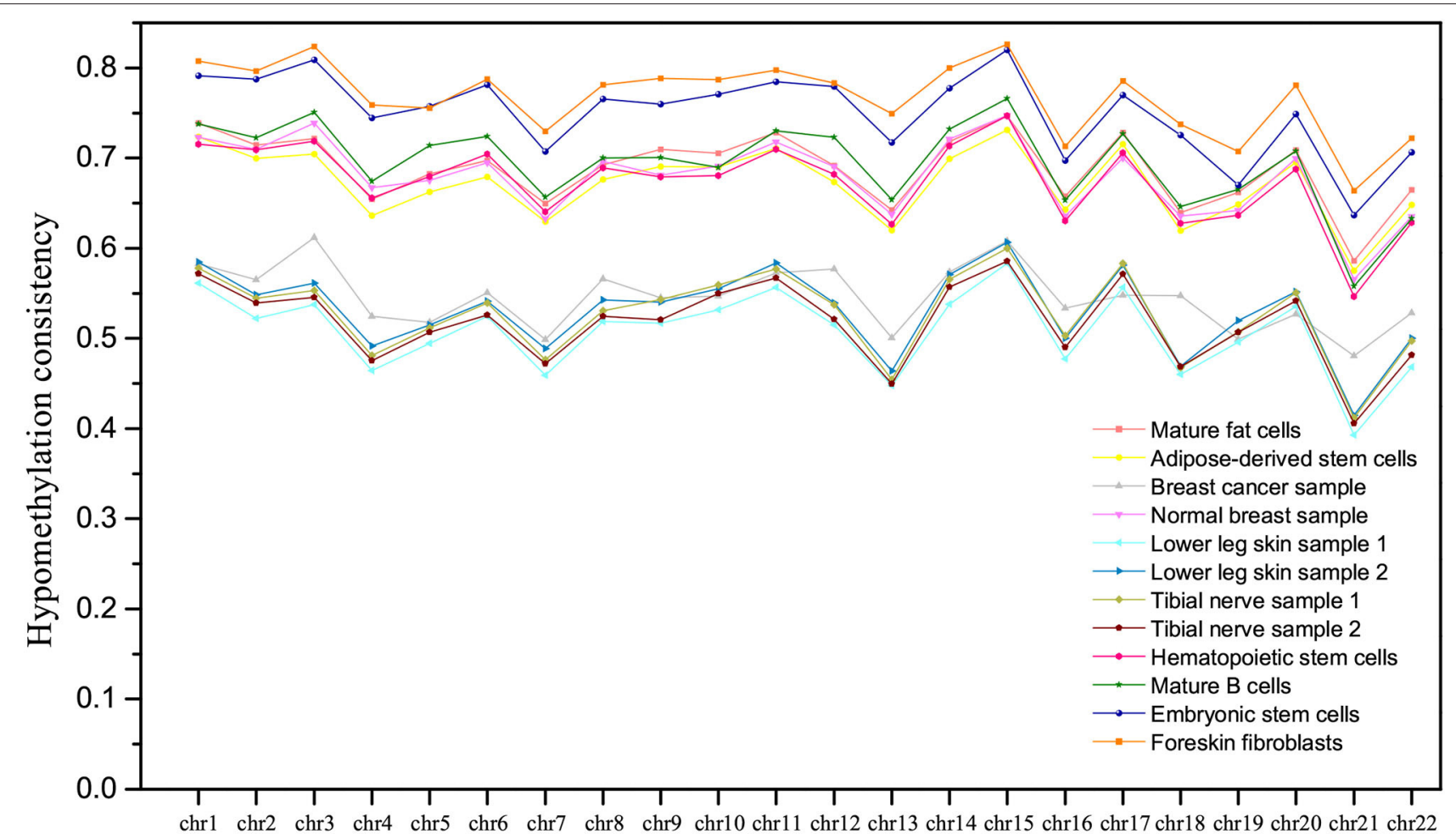

\section{Chromosome}

FIGURE 5 | The comparison of hypomethylation consistency of homologous chromosomes in different tissues/cell types.

TABLE 3 | Four types of DMRs identified by MHap_DMR for each pair of samples.

\begin{tabular}{|c|c|c|c|c|}
\hline Pairs of samples & $\begin{array}{l}\text { Type } 1 \text { DMR } \\
\text { (hypo- } \\
\text { vs. } \\
\text { non-hypo) }\end{array}$ & $\begin{array}{c}\text { Type } 2 \text { DMR } \\
\text { (consistent hypo- } \\
\text { vs. } \\
\text { semi-hypo) }\end{array}$ & $\begin{array}{c}\text { Type 3 DMR } \\
\text { (consistent hyper- } \\
\text { vs. } \\
\text { semi-hyper) }\end{array}$ & $\begin{array}{c}\text { Type } 4 \text { DMR } \\
\text { with } \\
\text { other modes }\end{array}$ \\
\hline Mature fat cells & 1,156 & 1,032 & 583 & 309 \\
\hline vs. & (LL vs. HH: 1) & (LL vs. HL: 574) & & \\
\hline vs. & (LL vs. HH: 15,175) & (LL vs. HL: 1,351) & & \\
\hline Normal breast & (HL vs. HH: 650) & & & \\
\hline Hematopoietic stem cells & 1,468 & 625 & 182 & 257 \\
\hline vs. & (LL vs. HH: 391) & (LL vs. HL: 286) & & \\
\hline Mature B cells & (HL vs. HH: 223) & & & \\
\hline
\end{tabular}

cells vs. adipose-derived stem cells, embryonic stem cells (hESCs) vs. foreskin fibroblasts (hESC-Fibro cells), and mature B cells vs. hematopoietic stem cells. In this study, MHap_DMR reports the DMRs with $p<0.05$ and $M H D>0.5$.

Based on the MHMs of samples on DMRs, the identified DMRs can be further classified into four groups:
1. hypomethylation mode (a MHM containing L) vs. non-hypomethylation mode (a MHM not containing $L)$; 2. hypomethylation consistent mode $L L$ vs. semihypomethylation mode (an unconsistent MHM containing $L)$; 3. hypermethylation consistent mode $H H$ vs. semihypermethylation mode (an unconsistent MHM containing 
TABLE 4 | The number of DMRs identified by different methods.

\begin{tabular}{|c|c|c|c|c|c|}
\hline Pairs of samples & MHap_DMR & CpG_MPs & DMRCaller & S SMART & Metilene \\
\hline \multicolumn{6}{|l|}{ Mature fat cells } \\
\hline vs. & 3,080 & 932 & 4,081 & 2,152 & 44,359 \\
\hline \multicolumn{6}{|l|}{$\begin{array}{l}\text { Adipose-derived stem } \\
\text { cells }\end{array}$} \\
\hline \multicolumn{6}{|l|}{ Breast cancer } \\
\hline vs. & 21,489 & 233,298 & 861,108 & 353,565 & 357,980 \\
\hline \multicolumn{6}{|l|}{ Normal breast } \\
\hline \multicolumn{6}{|l|}{$\begin{array}{l}\text { Hematopoietic stem } \\
\text { cells }\end{array}$} \\
\hline vs. & 2,532 & 26,526 & 172,475 & 50,453 & 75,180 \\
\hline \multicolumn{6}{|l|}{ Mature B cells } \\
\hline \multicolumn{6}{|l|}{ Embryonic stem cells } \\
\hline vs. & 10,922 & 130,376 & 385,877 & 282,617 & 338,631 \\
\hline Foreskin fibroblasts & & & & & \\
\hline
\end{tabular}

H); 4. DMR with other modes. The number of these types of DMRs between each pair of samples is listed in Table 3.

To investigate the methylation changing directions at the methylation haplotype level, the number of some subtypes of DMRs in Type 1 and Type 2 DMRs is specified. For example, in Type 1 DMRs, the number of DMRs with hypomethylation consistent mode $L L$ vs. hypermethylation consistent mode $H H$ and the number of DMRs with hypomethylation unconsistent mode $H L$ vs. hypermethylation consistent mode $H H$ are listed.

In Type 1 DMRs, it can be observed that there is only $1 \mathrm{DMR}$ with hypomethylation consistent mode $L L$ vs. hypermethylation mode consistent $\mathrm{HH}$ in mature fat cells and adipose-derived stem cells. It may indicate that the methylation statuses of two homologous chromosomes are seldom changed simultaneously during the differentiation from adipose-derived stem cells to mature fat cells.

In Type 1 DMRs between breast cancer and normal breast, it can be observed that there are 13,173 DMRs with hypermethylation consistent mode $H H$ in breast cancer and hypomethylation consistent mode $L L$ in normal breast, while there are only 2,002 DMRs with hypomethylation consistent mode $L L$ in breast cancer and hypermethylation consistent mode $H H$ in normal breast. It suggests that many regions with hypomethylation consistent mode $L L$ in normal breast become hypermethylated in breast cancer, while a small quantity of regions with hypermethylation consistent mode $\mathrm{HH}$ in normal breast become hypomethylated in breast cancer. Further, comparing the number of four types of DMRs between breast cancer and normal breast, it may indicate that, in breast cancer, the methylation statuses of homologous chromosomes changes in the same direction (hypomethylated or hypermethylated) simultaneously in many cases. The MHMs of DMRs among different samples can indicate the methylation changing directions of homologous chromosomes in cell differentiation and cancerization.

\subsection{Compared With Comparative Methods}

To further demonstrate the performance of MHap_DMR, four comparative tools were also applied to these four pairs of samples, including CpG_MPs (Su et al., 2012), DMRCaller (Catoni et al., 2018), SMART (Liu et al., 2015), and Metilene (Jühling et al., 2016). The default parameter settings were adopted when running these methods.

The numbers of DMRs identified by different methods are compared, as shown in Table 4. Metilene always predicts a larger number of DMRs with low methylation level differences than other methods. MHap_DMR predicts a smaller number of DMRs than other methods, because it works on candidate regions predefined on the $\mathrm{CpG}$ dense regions. All the methods report a largest number of DMRs between breast cancer sample and normal breast sample, and a second largest number of DMRs between embryonic stem cells and foreskin fibroblasts. This consistency indicates that DNA methylation is altered a lot in cancerization, and the methylation difference between embryonic stem cells and foreskin fibroblasts is larger than that between other types of stem cells and the cells derived from these stem cells.

\section{CONCLUSION}

In this paper, MHap is developed to construct methylation haplotypes for homologous chromosomes in $\mathrm{CpG}$ dense regions. Through the analysis based on methylation haplotypes of homologous chromosomes, we found that majority of methylation haplotypes are consistent between homologous autosomes, while a lower methylation consistency was observed in the breast cancer sample. Further, the hypomethylation consistency of derived cells is higher than that of the corresponding undifferentiated stem cells. The hypomethylation consistency can be used as a feature for cell clustering. DMRs identified by MHap_DMR based on methylation haplotypes can help to investigate the methylation changing directions of homologous chromosomes in cell differentiation and cancerization.

\section{DATA AVAILABILITY STATEMENT}

Publicly available datasets were analyzed in this study. This data can be found at: the ENCODE project (https:// www.encodeproject.org/) through the access sample ids ENCSR930WUY, ENCSR128RMY, ENCSR752OCM, and ENCSR658MZU, the GEO database (https://www.ncbi.nlm. nih.gov/geo/) through GEO accession numbers GSE29069, GSE19418, and GSE31971, and the NCBI SRA database (https://www.ncbi.nlm.nih.gov/sra) under the accession number SRA0238292. 


\section{AUTHOR CONTRIBUTIONS}

$\mathrm{XP}$ and XD conceived and designed the approach. XP and YL performed the experiments. YL and XK analyzed the data. XP wrote the manuscript. XP and XZ supervised the whole study process and revised the manuscript. All authors have read and approved the final version of manuscript.

\section{REFERENCES}

Abante, J., Fang, Y., Feinberg, A., and Goutsias, J. (2020). Detection of haplotypedependent allele-specific DNA methylation in WGBS data. Nat. Commun. 11, 1-13. doi: 10.1038/s41467-020-19077-1

Baylin, S. B. (2005). DNA methylation and gene silencing in cancer. Nat. Rev. Clin. Oncol. 2:S4. doi: 10.1038/ncponc0354

Catoni, M., Tsang, J. M., Greco, A. P., and Zabet, N. R. (2018). DMRcaller: a versatile $\mathrm{R} /$ bioconductor package for detection and visualization of differentially methylated regions in $\mathrm{CpG}$ and non-CpG contexts. Nucl. Acids Res. 46:e114. doi: 10.1093/nar/gky602

Chen, Q., Lai, D., Lan, W., Wu, X., Chen, B., Chen, Y.-P. P., et al. (2019). ILDMSF: inferring associations between long non-coding RNA and disease based on multi-similarity fusion. IEEE/ACM Trans. Comput. Biol. Bioinformatics. 18, 1106-1112 doi: 10.1109/TCBB.2019.2936476

Cheung, W. A., Shao, X., Morin, A., Siroux, V., Kwan, T., Ge, B., et al. (2017). Functional variation in allelic methylomes underscores a strong genetic contribution and reveals novel epigenetic alterations in the human epigenome. Genome Biol. 18, 1-21. doi: 10.1186/s13059-017-1173-7

Condon, D. E., Tran, P. V., Lien, Y.-C., Schug, J., Georgieff, M. K., Simmons, R. A., et al. (2018). Defiant:(dmrs: easy, fast, identification and annotation) identifies differentially methylated regions from iron-deficient rat hippocampus. $B M C$ Bioinformatics 19:31. doi: 10.1186/s12859-018-2037-1

Eden, A., Gaudet, F., Waghmare, A., and Jaenisch, R. (2003). Chromosomal instability and tumors promoted by DNA hypomethylation. Science 300:455. doi: $10.1126 /$ science. 1083557

Feng, H., Conneely, K. N., and Wu, H. (2014). A Bayesian hierarchical model to detect differentially methylated loci from single nucleotide resolution sequencing data. Nucl. Acids Res. 42:e69. doi: 10.1093/nar/gku154

Gomez, L., Odom, G. J., Young, J. I., Martin, E. R., Liu, L., Chen, X., et al. (2019). coMethDMR: accurate identification of co-methylated and differentially methylated regions in epigenome-wide association studies with continuous phenotypes. Nucl. Acids Res. 47:e98. doi: 10.1093/nar/gkz590

Goto, T., and Monk, M. (1998). Regulation of x-chromosome inactivation in development in mice and humans. Microbiol. Mol. Biol. Rev. 62, 362-378. doi: 10.1128/MMBR.62.2.362-378.1998

Hansen, K. D., Langmead, B., and Irizarry, R. A. (2012). BSmooth: from whole genome bisulfite sequencing reads to differentially methylated regions. Genome Biol. 13:R83. doi: 10.1186/gb-2012-13-10-r83

Hebestreit, K., Dugas, M., and Klein, H.-U. (2013). Detection of significantly differentially methylated regions in targeted bisulfite sequencing data. Bioinformatics 29, 1647-1653. doi: 10.1093/bioinformatics/btt263

Hodges, E., Molaro, A., Dos Santos, C. O., Thekkat, P., Song, Q., Uren, P. J., et al. (2011). Directional DNA methylation changes and complex intermediate states accompany lineage specificity in the adult hematopoietic compartment. Mol. Cell 44, 17-28. doi: 10.1016/j.molcel.2011.08.026

Hon, G. C., Hawkins, R. D., Caballero, O. L., Lo, C., Lister, R., Pelizzola, M., et al. (2012). Global DNA hypomethylation coupled to repressive chromatin domain formation and gene silencing in breast cancer. Genome Res. 22, 246-258. doi: $10.1101 /$ gr.125872.111

Hu, X., Li, M., Wang, L., Li, X., Wu, F.-X., and Wang, J. (2019). "Classification of schizophrenia by iterative random forest feature selection based on DNA methylation array data," in 2019 IEEE International Conference on Bioinformatics and Biomedicine (BIBM) (San Diego, CA), 807-811. doi: 10.1109/BIBM47256.2019.8983308

Jühling, F., Kretzmer, H., Bernhart, S. H., Otto, C., Stadler, P. F., and Hoffmann, S. (2016). metilene: Fast and sensitive calling of differentially

\section{FUNDING}

This work was supported by the National Natural Science Foundation of China (Nos. 61702555, 61662028, and U1909208), the National Key R\&D Program of China (No. 2018YFC0910504), 111 Project (No. B18059), and Hunan Provincial Science and Technology Program (2018WK4001).

methylated regions from bisulfite sequencing data. Genome Res. 26, 256-262. doi: 10.1101/gr.196394.115

Krueger, F., and Andrews, S. R. (2011). Bismark: a flexible aligner and methylation caller for bisulfite-seq applications. Bioinformatics 27, 1571-1572. doi: 10.1093/bioinformatics/btr167

Lan, W., Lai, D., Chen, Q., Wu, X., Chen, B., Liu, J., et al. (2020). LDICDL: LncRNA-disease association identification based on collaborative deep learning. IEEE/ACM Trans. Comput. Biol. Bioinformatics. doi: 10.1109/TCBB.2020.3034910

Laurent, L., Wong, E., Li, G., Huynh, T., Tsirigos, A., Ong, C. T., et al. (2010). Dynamic changes in the human methylome during differentiation. Genome Res. 20, 320-331. doi: 10.1101/gr.101907.109

Lea, A. J., Tung, J., and Zhou, X. (2015). A flexible, efficient binomial mixed model for identifying differential DNA methylation in bisulfite sequencing data. PLoS Genet. 11:e1005650. doi: 10.1371/journal.pgen. 1005650

Lister, R., Pelizzola, M., Kida, Y. S., Hawkins, R. D., Nery, J. R., Hon, G., et al. (2011). Hotspots of aberrant epigenomic reprogramming in human induced pluripotent stem cells. Nature 471:68. doi: 10.1038/nature09798

Liu, H., Liu, X., Zhang, S., Lv, J., Li, S., Shang, S., et al. (2015). Systematic identification and annotation of human methylation marks based on bisulfite sequencing methylomes reveals distinct roles of cell type-specific hypomethylation in the regulation of cell identity genes. Nucl. Acids Res. 44, 75-94. doi: 10.1093/nar/gkv1332

Lokk, K., Modhukur, V., Rajashekar, B., Märtens, K., Mägi, R., Kolde, R., et al. (2014). DNA methylome profiling of human tissues identifies global and tissue-specific methylation patterns. Genome Biol. 15:3248. doi: 10.1186/gb-2014-15-4-r54

Mohandas, T., Sparkes, R., and Shapiro, L. (1981). Reactivation of an inactive human $\mathrm{x}$ chromosome: evidence for $\mathrm{x}$ inactivation by DNA methylation. Science 211, 393-396. doi: 10.1126/science.6164095

Ni, P., Huang, N., Zhang, Z., Wang, D.-P., Liang, F., Miao, Y., et al. (2019). DeepSignal: detecting DNA methylation state from nanopore sequencing reads using deep-learning. Bioinformatics 35, 4586-4595. doi: 10.1093/bioinformatics/btz276

Park, Y., Figueroa, M. E., Rozek, L. S., and Sartor, M. A. (2014). Methylsig: a whole genome DNA methylation analysis pipeline. Bioinformatics 30, 2414-2422. doi: 10.1093/bioinformatics/btu339

Park, Y., and Wu, H. (2016). Differential methylation analysis for BS-seq data under general experimental design. Bioinformatics 32, 1446-1453. doi: 10.1093/bioinformatics/btw026

Scott, C. A., Duryea, J. D., MacKay, H., Baker, M. S., Laritsky, E., Gunasekara, C. J., et al. (2020). Identification of cell type-specific methylation signals in bulk whole genome bisulfite sequencing data. Genome Biol. 21, 1-23. doi: 10.1186/s13059-020-02065-5

Stockwell, P. A., Chatterjee, A., Rodger, E. J., and Morison, I. M. (2014). DMAP: differential methylation analysis package for RRBS and WGBS data. Bioinformatics 30, 1814-1822. doi: 10.1093/bioinformatics/btu126

Su, J., Yan, H., Wei, Y., Liu, H., Liu, H., Wang, F., et al. (2012). CpG_MPs: identification of CPG methylation patterns of genomic regions from high-throughput bisulfite sequencing data. Nucl. Acids Res. 41:e4. doi: $10.1093 / \mathrm{nar} / \mathrm{gks} 829$

Sun, S., and Yu, X. (2016). Hmm-fisher: identifying differential methylation using a hidden Markov model and fisher's exact test. Stat. Appl. Genet. Mol. Biol. 15, 55-67. doi: 10.1515/sagmb-2015-0076

The ENCODE Project Consortium (2012). An integrated encyclopedia of DNA elements in the human genome. Nature 489:57. doi: 10.1038/nature11247 
Wang, Z., Li, X., Jiang, Y., Shao, Q., Liu, Q., Chen, B., et al. (2015). swDMR: a sliding window approach to identify differentially methylated regions based on whole genome bisulfite sequencing. PLOS ONE 10:e0132866. doi: 10.1371/journal.pone.0132866

Wen, Y., Chen, F., Zhang, Q., Zhuang, Y., and Li, Z. (2016). Detection of differentially methylated regions in whole genome bisulfite sequencing data using local Getis-Ord statistics. Bioinformatics 32, 3396-3404. doi: 10.1093/bioinformatics/btw497

Wu, H., Xu, T., Feng, H., Chen, L., Li, B., Yao, B., et al. (2015). Detection of differentially methylated regions from whole-genome bisulfite sequencing data without replicates. Nucl. Acids res. 43:e141. doi: 10.1093/nar/ gkv715

Xiaoqing, P., Hong-Dong, L., Fang-Xiang, W., and Jianxin, W. (2020). Identifying the tissues-of-origin of circulating cell-free DNAs is a promising way in noninvasive diagnostics. Brief. Bioinformatics 22:bbaa060. doi: 10.1093/bib/bbaa060

Xu, Z., Xie, C., Taylor, J. A., and Niu, L. (2020). ipDMR: identification of differentially methylated regions with interval p-values. Bioinformatics 37, 711-713. doi: 10.1093/bioinformatics/btaa732

Yan, C., Duan, G., Wu, F.-X., Pan, Y., and Wang, J. (2019). BRWMDA: predicting microbe-disease associations based on similarities and bi-random walk on disease and microbe networks. IEEE/ACM Trans. Comput. Biol. Bioinformatics 17, 1595-1604. doi: 10.1109/TCBB.2019.2907626
Yan, C., Duan, G., Wu, F.-X., Pan, Y., and Wang, J. (2021). MCHMDA: Predicting microbe-disease associations based on similarities and low-rank matrix completion. IEEE/ACM Trans. Comput. Biol. Bioinformatics 18, 611-620. doi: 10.1109/TCBB.2019.2926716

Yan, C., Wang, J., Ni, P., Lan, W., Wu, F.-X., and Pan, Y. (2017). DNRLMF-MDA: predicting microRNA-disease associations based on similarities of microRNAs and diseases. IEEE/ACM Trans. Comput. Biol. Bioinformatics 16, 233-243. doi: 10.1109/TCBB.2017.2776101

Yan, C., Wang, J., and Wu, F.-X. (2018). DWNN-RLS: regularized least squares method for predicting circRNA-disease associations. BMC Bioinformatics 19(Suppl. 19):520. doi: 10.1186/s12859-018-2522-6

Conflict of Interest: The authors declare that the research was conducted in the absence of any commercial or financial relationships that could be construed as a potential conflict of interest.

Copyright (๑ 2021 Peng, Li, Kong, Zhu and Ding. This is an open-access article distributed under the terms of the Creative Commons Attribution License (CC BY). The use, distribution or reproduction in other forums is permitted, provided the original author(s) and the copyright owner(s) are credited and that the original publication in this journal is cited, in accordance with accepted academic practice. No use, distribution or reproduction is permitted which does not comply with these terms. 